\title{
Chr1 95026203-ALK, a novel intergenic fusion identified in a patient with lung adenocarcinoma with nodular ground-glass opacity
}

\author{
Qiang Chen ${ }^{1 \#}$, Yan Yan ${ }^{2 \#}$, Chuanping Yang", Guangjun Liu ${ }^{1}$, Gaoming Wang ${ }^{1}$, Si Li ${ }^{3}$, Dongsheng Chen ${ }^{3}$, \\ Mingzhe $\mathrm{Xiao}^{3}$, Yanmin $\mathrm{Wu}^{4} \wedge$ \\ ${ }^{1}$ Department of Thoracic Surgery, Xuzhou Central Hospital, Xuzhou, China; ${ }^{2}$ Department of Cardiology, The Affiliated Hospital of Xuzhou Medical \\ University, Xuzhou, China; ${ }^{3}$ The Medical Department, The State Key Lab of Translational Medicine and Innovative Drug Development, Nanjing \\ Simcere Medical Laboratory Science Co., Ltd., Jiangsu Simcere Diagnostics Co., Ltd., Jiangsu, China; ${ }^{4}$ Department of Respiratory and Critical Care \\ Medicine, Xuzhou Central Hospital, Xuzhou, China \\ \#These authors contributed equally to this work. \\ Correspondence to: Yanmin Wu. Department of Respiratory and Critical Care Medicine, Xuzhou Central Hospital, 199 South Jiefang Road, Xuzhou \\ 221009, China. Email: candycy1119@163.com.
}

Submitted Apr 19, 2021. Accepted for publication Jun 11, 2021.

doi: $10.21037 /$ jtd-21-689

View this article at: https://dx.doi.org/10.21037/jtd-21-689

The rearrangement of anaplastic lymphoma kinase $(A L K)$ gene accounts for $3-5 \%$ in non-small cell lung cancer (NSCLC) (1). Novel variants including intergenic fusion were widely analyzed since they did bring significant clinical benefit for the fusion carriers (2). Therefore, novel variants including intergenic fusions are getting attention to illustrate potential targetable variants. At the meantime, lung cancer with ground-glass opacity (GGO) component predicts better prognosis, while some of them could progress rapidly (3). Therefore, the molecular profile of GGO-featured lung cancer is under widely investigation to improve current understanding on it. Studies has revealed significantly lower frequency of $A L K$ fusion in patients presenting GGO, while the occurrence of $A L K$ fusion could predict higher tumor burden and more aggressive progression (4). Thus, reporting the potential targetable fusion variant might guide those patients' treatment management. Here we report a novel Chr1 95026203$A L K$ intergenic fusion, identified in a patient with lung adenocarcinoma with nodular GGO.

A 48-year-old male smoker went to our hospital for reexamination in September 2020 after discovering a pulmonary nodule 6 months ago. Chest computed tomography scan revealed an irregular-shaped nodule with GGO in localized apical and posterior segment of superior lobe of left lung and diffuse bilateral pulmonary nodules (Figure 1A,B,C,D). Multiple enlarged lymph nodes were observed in mediastinum and left neck. A slight pericardial effusion was also discovered. Pathological examination was conducted with the needle biopsy of the enlarged left cervical lymph node [hematoxylin and eosin (H\&E) staining in Figure 1E]. The immunohistochemistry (IHC) staining showed TTF-1(+), Napsin A(+), CK7(+), CK5/6(-), P63(+), Ki67(25\%+), P40(+), Syn- and PAS(+). The disease was diagnosed as metastatic lung adenocarcinoma (poorly differentiated, T1N3M1b).

To seek for individualized therapy, the formalinfixed and paraffin-embedded specimens were subjected to DNA-based next-generation sequencing (NGS) analysis [in College of American Pathologists-certified laboratory) with a panel of 9 genes including $E G E R, A L K$, ROS1, RET, MET, ERBB2, KRAS, BRAF, NTRK1/2/3. A novel Chr1 95026203-ALK intergenic fusion was detected (Figure 2A,B)]. This fusion was generated by

$\wedge$ ORCID: 0000-0001-8562-3546. 

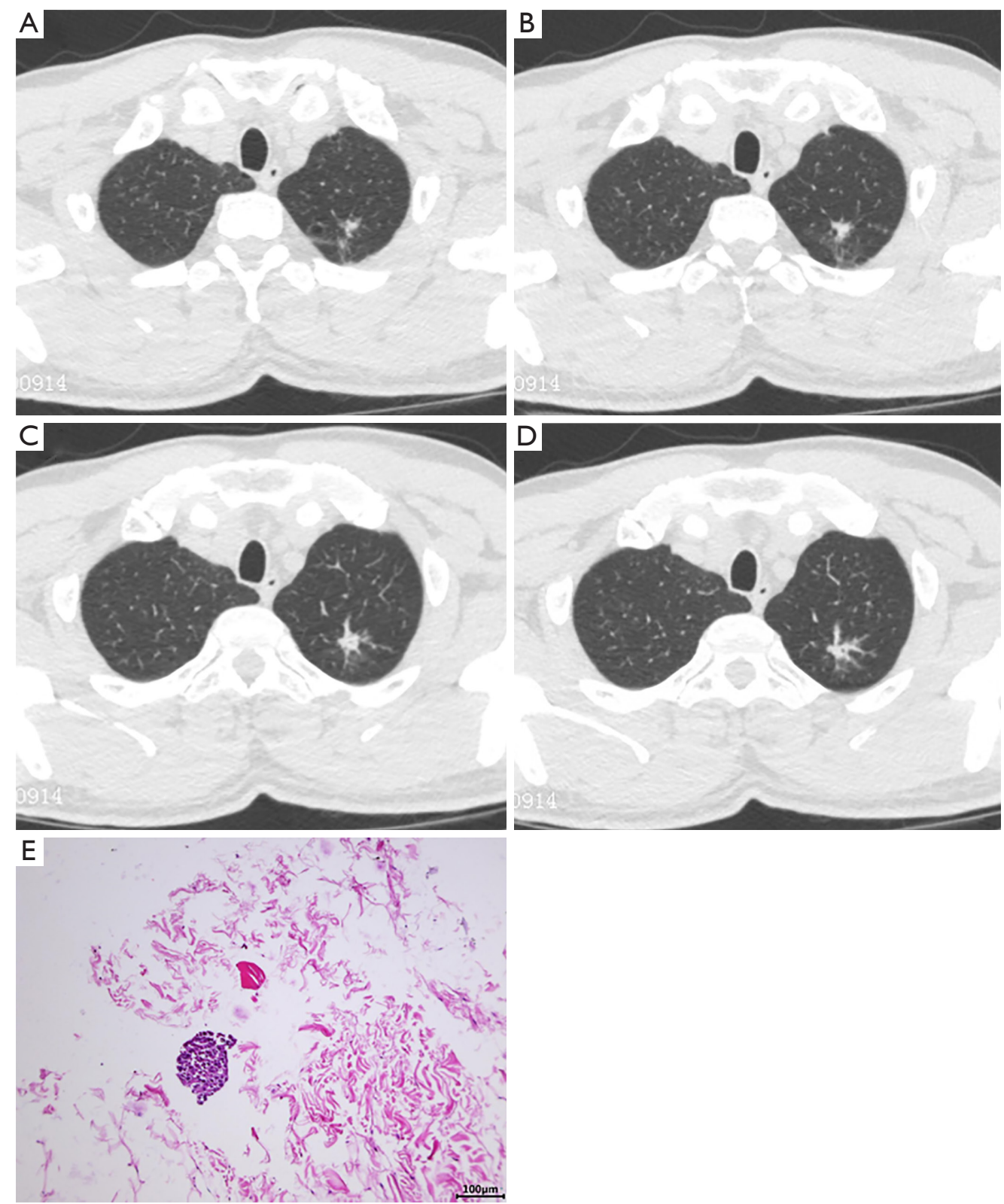

Figure 1 Diagnostic images of tumor lesions and hematoxylin and eosin (H\&E) staining. (A,B,C,D) Pulmonary lesions by chest computed tomography scan at diagnose on $14^{\text {th }}$ September, the images of $(A, B, C, D)$ are arranged from top-to-bottom direction. (E) H\&E staining of the biopsy specimen $(100 \times)$. H\&E, hematoxylin and eosin.

Chr1 95026203 (intergenic region between $F 3$ and $S L C 44 A 3)$ and exons 20-29 of $A L K$, with the abundance of $3.86 \%$. No concurrent alterations were detected. This novel fusion retained the whole kinase domain of $A L K$ and expression of the $A L K$ protein was confirmed by IHC analysis (3+ staining) (Figure 2C). The patient adopted chemotherapy (pemetrexed combined with lobaplatin) as first-line treatment eventually due to its lower expense and maintained stable disease after 1 cycle of treatment. According to last follow-up visit on 22 April 2021, no progression was observed after 6 cycle's treatment. The progression-free survival is more than 7 months. Figure 3 showed the varying trend of carcino-embryonic antigen (CEA). 

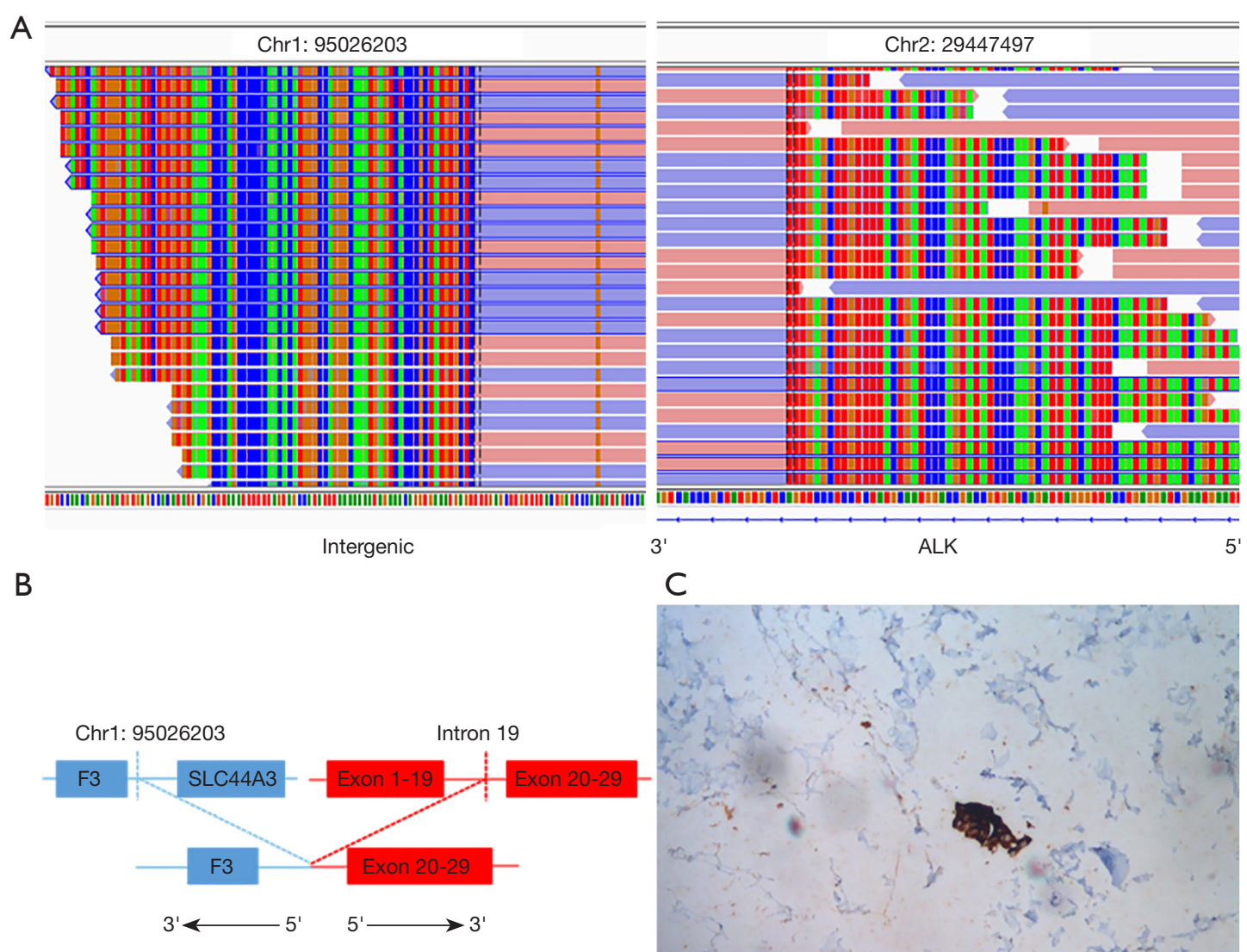

C

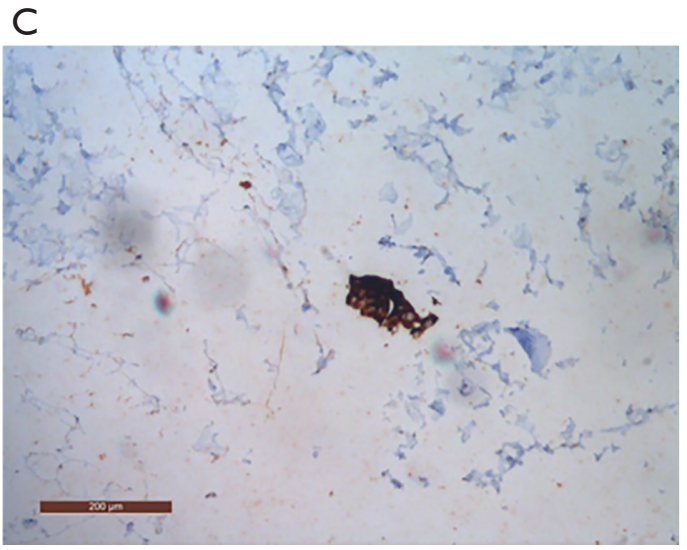

Figure 2 Detection of ALK fusion. (A) The integrative genomics viewer screenshot of Chr1 95026203-ALK fusion, detected by DNAbased NGS with specimen of cervical lymph node metastasis. (B) Schematic diagram of the detected fusion, this variant was generated by the fusion of intergenic region between F3 and SLC44A3 with exons 20-29 of ALK. (C) Positive anaplastic lymphoma kinase expression by IHC $(3+, 100 \times) . A L K$, anaplastic lymphoma kinase; NGS, next-generation sequencing; IHC, immunohistochemistry.

To our knowledge, this is the first report describing the fusion of Chr1 95026203 with $A L K$ in lung adenocarcinoma. Actually, methods for detecting fusions are widely discussed recently. DNA-based NGS was able to identify the specific partner genes and breakpoints, as well as the potential resistant mutations in $A L K$ or other concurrent gene alterations. Therefore, it could be a proper primary choice for patients. Generally, fusions compose of intergenic regions are theoretically unlikely to generate functional fusion transcript, while accumulating evidence suggests that intergenic fusion might also generate functional fusion protein after transcription (chromothripsis and alternative splicing as potential mechanism) (2). Therefore, further RNA-level or protein-level validation was often recommended to capture the possible actionable fusions and guide target therapy (2). In this case, $A L K \mathrm{kept}$ the whole kinase domain according to results of DNA-based NGS and $A L K$ fusion protein was demonstrated positive by IHC. This indicated fusion of Chr1 95026203-ALK might generate functional fusion protein and the patient might benefit from $A L K$ inhibitors when considering the following treatment.

Another key point is that this novel fusion was identified in a patient with GGO. To figure out the molecular profile of GGO-featured lung cancer, studies on driver gene like $E G F R$ and $A L K$ are increasing (5). Frequency of $A L K$ fusion ( $2.9 \%$ in lesion with pure GGO) was found significantly lower in GGO-featured lung cancer (6), leaving less 
3
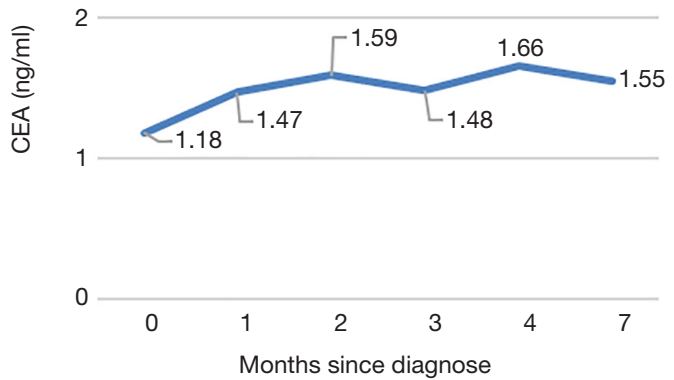

Figure 3 The level of CEA from diagnose in September 2020 to the last follow-up visit in April 2021. CEA, carcino-embryonic antigen.

opportunity of $A L K$ inhibitors for those patients. While fusion of $A L K$ is found associated with advanced stage and larger tumor size, suggesting its association with aggressive progression of lung adenocarcinoma. Therefore, it is important to identify potential targetable $A L K$ fusion variant and accordingly decide individualized management strategy. In this case, this novel fusion of Chr1 95026203$A L K$ was detected, although with lymph node metastasis, it is probably shared by the nodule with GGO, since a 98\% concordance of $A L K$ fusion between primary and lymph node metastasis was revealed (7). This might enrich the evidence of $A L K$ fusion in GGO-featured lung cancer and implied potential tumor progression trend and target therapy for this subgroup. Future studies on the molecular difference of tumor cells between GGO and solid lesions, as well as its impact on patients' tumor progression are also needed.

\section{Acknowledgments}

We thank Ran Ding, Guanghua Lu, Wanglong Deng and Chuang Qi from Jiangsu Simcere Diagnostics for the kindly assistance.

Funding: Young Medical Talents in Jiangsu Province (QNRC:2016381) and Training Project of Clinical Technical Experts in Xuzhou (2019GG018).

\section{Footnote}

Provenance and Peer Review: This article was a standard submission to the journal. The article has undergone external peer review.

Peer Review File: Available at https://dx.doi.org/10.21037/ jtd-21-689

Conflicts of Interest: All authors have completed the ICMJE uniform disclosure form (available at https://dx.doi. org/10.21037/jtd-21-689). The authors have no conflicts of interest to declare.

Ethical Statement: The authors are accountable for all aspects of the work in ensuring that questions related to the accuracy or integrity of any part of the work are appropriately investigated and resolved. All procedures performed in studies involving human participants were in accordance with the ethical standards of the institutional and national research committees and with the Helsinki Declaration (as revised in 2013).

Open Access Statement: This is an Open Access article distributed in accordance with the Creative Commons Attribution-NonCommercial-NoDerivs 4.0 International License (CC BY-NC-ND 4.0), which permits the noncommercial replication and distribution of the article with the strict proviso that no changes or edits are made and the original work is properly cited (including links to both the formal publication through the relevant DOI and the license). See: https://creativecommons.org/licenses/by-nc-nd/4.0/.

\section{References}

1. Solomon B, Varella-Garcia M, Camidge DR. ALK gene rearrangements: a new therapeutic target in a molecularly defined subset of non-small cell lung cancer. J Thorac Oncol 2009;4:1450-4.

2. Li W, Liu Y, Li W, et al. Intergenic Breakpoints Identified by DNA Sequencing Confound Targetable Kinase Fusion Detection in NSCLC. J Thorac Oncol 2020;15:1223-31.

3. Zhang Y, Fu F, Chen H. Management of Ground-Glass Opacities in the Lung Cancer Spectrum. Ann Thorac Surg 2020;110:1796-804.

4. Ko SJ, Lee YJ, Park JS, et al. Epidermal growth factor receptor mutations and anaplastic lymphoma kinase rearrangements in lung cancer with nodular ground-glass opacity. BMC Cancer 2014;14:312.

5. Gao JW, Rizzo S, Ma LH, et al. Pulmonary ground-glass opacity: computed tomography features, histopathology 
and molecular pathology. Transl Lung Cancer Res 2017;6:68-75.

6. Shi J, Gu W, Zhao Y, et al. Clinicopathological and Prognostic Significance of EML4-ALK Rearrangement in Patients with Surgically Resected Lung Adenocarcinoma: A Propensity Score Matching Study. Cancer Manag Res

Cite this article as: Chen Q, Yan Y, Yang C, Liu G, Wang G, Li S, Chen D, Xiao M, Wu Y. Chr1 95026203-ALK, a novel intergenic fusion identified in a patient with lung adenocarcinoma with nodular ground-glass opacity. J Thorac Dis 2021;13(7):4618-4622. doi: 10.21037/jtd-21-689
2020;12:589-98.

7. Hou L, Ren S, Su B, et al. High concordance of ALK rearrangement between primary tumor and paired metastatic lymph node in patients with lung adenocarcinoma. J Thorac Dis 2016;8:1103-11. 